\title{
Gerenciamento da Cadeia de Abastecimento de Hortifrutigranjeiros Frescos: uma Pesquisa Exploratória no Reino Unido(1)
}

\author{
Hélio Zanquetto Filho \\ Andrew Fearne \\ Nélio Domingues Pizzolato
}

\section{ResUMo}

Uma das principais justificativas para a implementação do gerenciamento da cadeia de suprimentos é o aumento dos benefícios obtidos pelas empresas que se relacionam dentro da cadeia. Este artigo procura, no primeiro momento, estabelecer a simetria entre os benefícios, identificados empiricamente, com aqueles identificados na literatura. No segundo momento, analisa as correlações entre estes benefícios e o nível de investimentos (capital e tempo gerencial) realizados. Em se tratando de pesquisa exploratória, realizou-se uma pesquisa amostral probabilística, composta por de 99 empresas. Como instrumento de pesquisa foram utilizados questionários semi-estruturados, compostos por questões fechadas, enviados no primeiro semestre de 2002 para os gerentes operacionais de cada empresa. Para tratamento dos dados foi utilizada análise multivariada. Os resultados evidenciaram a consistência teórica da literatura, uma vez que confirmaram os benefícios alcançados pelas empresas. Além disso, foi demonstrado que o investimento em tempo gerencial é mais relevante que o investimento em capital para a obtenção dos benéficos almejados.

Palavras-chave: benefícios; parceria; cadeia de suprimento; investimentos; alimentos frescos do RU.

\begin{abstract}
One of the most relevant justifications for the implementing of supply chain management is the benefit generated by supply chain partnerships. This paper is firstly concerned with the symmetry between the theoretical and empirical benefits. Secondly, it aims to analyse the correlation between the empirical benefits and the level of investment made by the suppliers (capital and managerial time). A survey was chosen as the empirical research strategy, capturing 99 questionnaires from the population database. The questionnaires were sent to the operation managers during the first semester of 2002. For the data analyses, multivariate tests were applied. The results show symmetry between the theoretical and empirical benefits. In addition, the benefits are greater when the firms invest more in their managerial time than in capital.
\end{abstract}

Key words: benefits; buyer-supplier partnership; supply chain; investment; UK fresh produce. 


\section{INTRODUÇÃO}

De acordo com Van Hoek (1998) o Gerenciamento da Cadeia de Suprimentos (GCS) é caracterizado pelo controle, baseado na rede e na integração dos processos em suas áreas funcionais e nas interfaces regionais e organizacionais. A transparência no processo de avaliação de desempenho na cadeia de suprimentos faz com que as atividades não estejam sob o controle direto de uma única empresa. As atividades (unidade componentes dos processos empresariais) podem ser medidas e controladas em nível de transparência não experimentado anteriormente. Assim sendo, esta pesquisa tem interesse nos benefícios que ocorrem nas interfaces do relacionamento entre empresas parceiras.

Desta forma, no presente artigo, têm-se dois objetivos principais. O primeiro, comparar os benefícios dos fornecedores da cadeia de hortifrutigranjeiros, identificados na pesquisa empírica, com aqueles identificados na literatura. $\mathrm{O}$ segundo, aprofundar o entendimento dos relacionamentos destes benefícios com o nível de investimentos realizados pelos fornecedores. Para isso foram formuladas três hipóteses.

H1: Os benefícios alcançados na cadeia de hortifrutigranjeiros no Reino Unido coincidem com aqueles identificados pela literatura.

H2: As empresas que estão investindo maior volume de capital nos relacionamentos com seus compradores-chaves intermediários estão obtendo mais benefícios que as empresas que estão investindo pouco.

H3: As empresas que estão investindo maior tempo gerencial nos relacionamentos com seus compradores-chaves intermediários estão obtendo mais benefícios que as empresas que estão investindo pouco.

Para a realização da pesquisa de campo foi estabelecida uma parceria com o Centre for Food Chain Research (CFCR), do Imperial College at Wye da Universidade de Londres. Esta escolha foi realizada, uma vez que se identificou que os distribuidores de frutas e vegetais do Reino Unido estão entre os mais sofisticados do mundo e que a demanda sobre os seus fornecedores, particularmente os fornecedores de bens com marcas próprias, tem feito com que esta indústria de alimentos esteja entre as mais eficientes e inovadoras (Fearne $\&$ Hughes, 2000).

Do ponto de vista econômico-financeiro, o gerenciamento da cadeia de 
abastecimento de hortifrutigranjeiros é extremamente relevante para o Brasil, considerando que o país está entre os três maiores produtores mundiais de frutas, com produção maior que 34 milhões de toneladas, gerando 4 milhões de empregos diretos e um PIB agrícola de US\$ 11 bilhões. A importância econômica e social citada conduz à necessidade de se entender como são desenvolvidas as melhores práticas gerenciais na cadeia de hortifrutigranjeiros nos países com estrutura comercial mais madura. A partir desta pesquisa, será possível desenvolver modelos avançados de gerenciamento da cadeia de abastecimento, tanto nos hortifrutigranjeiros quanto em outras cadeias de abastecimento no Brasil.

Para facilitar a apresentação e o entendimento deste artigo fez-se a seguinte estruturação. Na próxima seção apresenta-se a metodologia, dando atenção especial às estratégias de pesquisa e aos métodos estatísticos utilizados. Na seção seguinte são expostas as limitações da pesquisa. Nas três seções subseqüentes é feita a revisão bibliográfica e são formuladas as hipóteses. Na penúltima seção são apresentados os resultados estatísticos com as respectivas análises. Na oitava e última seção são apresentadas as conclusões.

\section{Contextualização e Referencial Teórico}

\section{Contextualização}

Nas últimas décadas, muitas empresas iniciaram um processo de reestruturação de suas estratégias competitivas, desenvolvendo o conceito de gerenciamento da cadeia de suprimentos. Um dos reflexos observados nesse novo posicionamento está no fato de a competição ocorrer entre cadeias de suprimentos. Esse novo modelo competitivo, onde as empresas competem por meio da organização de suas cadeias, é uma das premissas básicas das estratégias competitivas atuais. A premissa adotada determina que o relacionamento entre fornecedores e compradores seja um relacionamento de parceria. A parceria, dentre outras características, pressupõe relacionamentos de longo prazo e freqüentes.

Diante disso, considera-se que os acionistas atuais e os potenciais investidores necessitam de novos instrumentos para monitorar o desempenho não só das empresas, de forma isolada, mas também da cadeia como um todo. As empresas têm mais de um fornecedor e mais de um comprador intermediário ou clientes finais. Nesse caso, tanto os fornecedores quanto os compradores intermediários podem pertencer a mais de uma cadeia, tornando complexa a tarefa de estruturar um modelo de avaliação de desempenho da cadeia de suprimentos. É sabido que as ações desenvolvidas no conjunto da cadeia se refletem direta ou indiretamente 
no desempenho das organizações. Assim, torna-se necessário criar um modelo de avaliação de desempenho da cadeia de forma tal que a cadeia seja considerada uma entidade organizacional conjunta e não um conjunto de entidades organizacionais. Entretanto esta nova estrutura só será possível, se ambos os parceiros dentro do GCS percebem ganhos por meio dos benefícios advindos desta relação ganha-ganha.

Na última década a competição entre cadeias de suprimentos vem se tornando uma realidade nas cadeias de alimentos devido às restrições de tempo de prateleira (perecibilidade dos produtos) e do crescimento da atenção dos consumidores com questões de segurança ambiental e alimentar (ex: alimentos geneticamente modificados, uso de agrotóxicos e bem-estar animal). Essa mudança de comportamento tem ocorrido mais fortemente nos países desenvolvidos e em grupos específicos nos países em desenvolvimento. No caso dos países da Comunidade Européia, o crescimento do GCS tem sido intensificado pelo aumento da competição, com a abertura dos mercados, o desenvolvimento demográfico e o incremento no uso das tecnologias de informação e comunicação (Fearne \& Hughes, 1998).

De acordo com Cavinato (1992), a competitividade na cadeia de suprimentos depende da cooperação entre as empresas para o desenvolvimento de produtos, produção e distribuição que conduz à redução de custos e inovação. Essa parceria requer uma visão e comportamento que enxerguem as vantagens totais para a firma e para a cadeia como um todo. A globalização aumentou as incertezas para as operações das empresas. A necessidade de lidar com tais incertezas vem conduzindo a um aumento considerável, tanto dos estoques quanto dos lead-time ao longo da cadeia de suprimentos (Bhatnagar \& Viswanathan, 2000).

O problema de implementação do gerenciamento da cadeia de suprimentos envolve a identificação dos membros da cadeia com os quais os processos-chaves serão desenvolvidos, que processos precisam ser conectados com cada um dos membros e que tipo ou nível de integração será utilizado em cada um dos processos interconectados. O objetivo do gerenciamento da cadeia de suprimentos é maximizar a competitividade e a rentabilidade das empresas e também de toda a rede de cadeias, incluindo-se, nesse caso, o consumidor final.

\section{Parcerias na Cadeia de Suprimentos}

O gerenciamento da cadeia de suprimentos expandiu as potencialidades das empresas na coordenação das atividades com relação a fornecimento, produção e entrega, dentro do conceito de colaboração com os parceiros pertencentes a cada canal de negócio (Stank \& Crum, 1999). De acordo com Hall e Adriani 
(1998), uma das razões fundamentais para que as empresas se insiram nas estratégias de parceria é a crença de que tais parcerias possuem a capacidade de criar novas vantagens competitivas que, de outra forma, seriam muito difíceis desenvolver isoladamente. De acordo com Gunasekaran, Patel e Tirtiroglu (2001), as parcerias devem estar voltadas diretamente para a associação de longo prazo entre as empresas, encorajando o desenvolvimento de esforços para se realizar planejamento e resolução de problemas conjuntamente.

No nível operacional, a coordenação do fornecimento, da produção e das atividades logísticas conjuntas muda a estrutura de funcionamento da cadeia com empresas isoladas para empresas coordenadas, com o objetivo de melhorar suas eficiências operacionais e aumentar a competitividade por meio da redução do tempo de atendimento das necessidades (Stank \& Crum, 1999). Conforme afirma Sandy (1999), os compradores e fornecedores são funcionalmente independentes, porém financeiramente dependentes. Em vez de estar atenta apenas aos preços, a empresa está mais preocupada com seus fornecedores para trabalhar cooperativamente, melhorando os serviços e inovando a tecnologia e o projeto de produtos (Maloni \& Benton, 1997).

\section{Formulação das Hipóteses}

\section{Hipótese H1}

Bowersox et al. (2000) afirmam que, a menos que os gerentes possam quantificar os benefícios operacionais e financeiros das suas iniciativas em direção à estruturação em forma de cadeia de suprimentos, os diretores e os acionistas das empresas hesitarão em apoiar suas iniciativas de implementar relacionamentos de parcerias com os fornecedores e consumidores intermediários.

Segundo Cooper et al. (1997), o gerenciamento da cadeia de suprimentos exige muito mais esforço, coordenação e análise do que as abordagens de gerenciamento de canais tradicionais. Os benefícios que as empresas esperam obter no futuro, ou que já estão obtendo, é o principal fator motivador das parcerias nas cadeias de suprimentos. De acordo com Lambert, Emmelhainz e Gardner (1996), não há dúvida de que existe diferença entre os benefícios alcançados tanto pelos fornecedores quanto pelos compradores; porém isso não é o mais relevante. $\mathrm{O}$ que interessa, realmente, é que ambas as partes obtenham benefícios que julguem relevantes, de forma tal que proporcionem a ambas as partes uma expectativa realista da força que a parceria pode ter em seus negócios.

O consenso nos relacionamentos dentro das cadeias de suprimentos é de que há uma conexão estrita entre os participantes da cadeia com objetivo de obter vantagens 
no mercado, acesso a novos produtos, e partilhamento e construção de novas oportunidades de negócios (Gunasekaran, Patel, \& Tirtiroglu, 2001). Outros aspectoschaves para a parceria incluem o nível de assistência mínima ao parceiro proporcionada por ambas as partes, a flexibilidade demonstrada por eles em relação aos requerimentos não programados anteriormente e o comprometimento mútuo que, potencialmente, melhora o benefício global do relacionamento.

Alguns resultados dessas parcerias são: o crescimento da rentabilidade, a melhoria nos processos e a melhoria na competitividade com relação à participação no mercado. Para o desenvolvimento do modelo de relacionamento entre parcerias, quatro benefícios potenciais para os fornecedores foram identificados, quais sejam: eficiência nos custos e uso dos ativos; melhorias no serviço prestado ao consumidor; vantagens de mercado; e melhoria na rentabilidade e crescimento nas vendas.

\section{Eficiência nos Custos e Uso dos Ativos}

Os ativos na cadeia de suprimentos incluem: contas a receber, plantas industriais, propriedades, equipamentos e estoques. Diante desse quadro, uma análise que se faz essencial é a da determinação da relação específica de associação entre custos e ativos. A informação obtida dessa relação deve ser combinada com o faturamento, de forma que se possa verificar quanto a relação afeta o retorno total obtido pelas empresas. No que diz respeito à melhoria da eficiência operacional e à conseqüiente melhoria de eficiências nos custos, esta pode ser alcançada com a melhoria nos processos internos da empresa, estendendo-se posteriormente esse esforço de melhoria aos fornecedores ou consumidores, ampliando assim a oportunidade de aumento nos ganhos obtidos pela empresa. Várias oportunidades de melhorias dentro da cadeia de suprimentos são factíveis, quando interessam às empresas parceiras (Gunasekaran et al., 2001). Como complemento, Stank e Crum (1999) observam que a integração entre as atividades pode conduzir à redução nos custos de transportes, manuseio, empacotamento, processamento de informação e, enfim, no custo final do produto, além de melhorar a eficiência operacional.

Fatores como o aumento no retorno sobre os ativos, redução do custo unitário, das perdas, no tempo de processamento e no custo por cliente são incentivos que têm sido utilizados para o desenvolvimento de parcerias entre as organizações (Oliver, 1990). Entretanto Gunasekaran et al. (2001) comentam o fato de que muitas empresas ainda não estão atentas ao relacionamento entre o gerenciamento do suprimento e a competitividade da empresa. Segundo eles, as empresas têm enfatizado apenas a redução de custos, em detrimento da melhoria da qualidade, serviços ao consumidor e acessos a novos recursos produtivos. 


\section{Melhorias no Serviço Prestado ao Consumidor}

Integrar as funções dentro da cadeia de suprimentos, por meio do desenvolvimento de parcerias, pode conduzir à melhoria dos serviços prestados, tanto ao consumidor intermediário quanto ao cliente final, seja recorrendo à redução de estoques, ao ciclo de produção, à melhoria nas informações relativas ao tempo de processamento e precisão. O gerenciamento da cadeia de suprimentos tem sido importante para estabelecer conexão com a melhoria de desempenho no serviço prestado ao consumidor (Stank \& Crum, 1999). Apesar de poder parecer uma conseqüência do funcionamento dos processos empresariais, o serviço ao cliente, quando estabelecido junto às metas das parcerias, é controlado por meio de indicadores de desempenho apropriados e adaptados para esse fim.

\section{Vantagens de Mercado}

Oliver (1990) argumenta que a integração forte de duas organizações pode alterar, para melhor, o mix de mercado das empresas e promover uma entrada facilitada em outros mercados. As alianças estratégicas são consideradas importantes para que se criem vantagens de mercado competitivas. Essas alianças originam-se da integração da cadeia, com a identificação das competências das parcerias, utilizando-as na construção das vantagens competitivas.

\section{Melhoria na Rentabilidade e Crescimento nas Vendas}

O benefício de melhoria na rentabilidade das empresas é, sem dúvida, um dos direcionadores mais importantes dentro da cadeia de suprimentos. A otimização da rentabilidade pode ser conseguida com o comprometimento de um aumento no volume comercializado, com a redução na variabilidade nas vendas, com o uso conjunto dos ativos, com redução no nível de estoques e outras melhorias (Noordewier, John, \& Nevin, 1990). Com base nesta revisão teórica a hipótese H1 foi formulada.

H1: Os benefícios alcançados na cadeia de hortifrutigranjeiros no Reino Unido coincidem com aqueles identificados pela literatura.

\section{Hipóteses $\mathrm{H} 2$ e $\mathrm{H3}$}

De acordo com Fearne e Hughes (1998), os pequenos fornecedores devem desenvolver um relacionamento colaborativo com seus parceiros, com o objetivo de participar do grupo de empresas que está obtendo os benefícios por meio da estratégia de adicionar valor aos produtos e serviços. Para estabelecer as parcerias, é necessário que os parceiros, entre eles o fornecedor, direcionem seus escassos 
recursos para os relacionamentos que consideram mais relevantes do ponto de vista estratégico. No caso específico identificado nesta pesquisa, considera-se que os fornecedores que investirem maior quantidade de recursos em seus compradores-chaves intermediários têm mais chances de obter benefícios consistentes do que se não investissem em suas parcerias. Assim, as duas hipóteses restantes foram formuladas.

H2: As empresas que estão investindo maior volume de capital nos relacionamentos com seus compradores-chaves intermediários estão obtendo mais benefícios que as empresas que estão investindo pouco.

H3: As empresas que estão investindo maior tempo gerencial nos relacionamentos com seus compradores-chaves intermediários estão obtendo mais benefícios que as empresas que estão investindo pouco.

\section{Procedimentos Metodológicos}

De acordo com a taxonomia apresentada por Vergara (1998), a presente pesquisa pode ser classificada, quanto aos seus fins, como descritiva e aplicada; e, quanto aos meios, como pesquisa de campo e empírica (com fontes primárias). $\mathrm{Na}$ estratégia de pesquisa amostral, as informações devem ser coletadas de uma amostra representativa da população. Os respondentes, normalmente, pertencem a categorias preestabelecidas, o que permite que as informações sejam estatisticamente analisadas.

Para o desenvolvimento do presente trabalho utilizou-se a pesquisa amostral, que apresentava o conjunto de características que melhor atendia às necessidades da pesquisa a ser realizada, utilizando como instrumento um questionário ${ }^{(2)}$ enviado por meio postal. A população foi composta por fornecedores de hortifrutigranjeiros ${ }^{(3)}$ que trabalhavam junto às empresas distribuidoras. Os grandes mercados atacadistas não foram incluídos na pesquisa, porquanto, mesmo sendo fornecedores dos distribuidores, funcionam, essencialmente, como empresas distribuidoras, uma vez que também vendem diretamente aos clientes finais.

Decidiu-se que a população pesquisada seria aquela com faturamento anual acima de $£ 1.000 .000,00$, algo próximo a $\mathrm{R} \$ 4.000 .000,00^{(4)}$, a fim de reduzir a heterogeneidade das empresas participantes, evitando assim a participação daquelas que fornecem produtos temporariamente (aproveitamento da sazonalidade característica do setor), e daquelas que lidam com os pequenos distribuidores locais. A homogeneidade foi definida com base nos argumentos 
apresentados por Heide e John (1990) de que a restrição por meio da homogeneização minimiza a influência de fatores externos que acarretam variação nas análises dos dados.

Desta forma, identificou-se que 308 empresas compunham, no total, a população. Os dados cadastrais das empresas foram obtidos a partir de publicações relacionadas ao setor, editadas no Reino Unido. Os principais documentos utilizados foram o Plimsoll Report (1999), o Food Trades Directory of the UK and Europe (2000) e três bases de dados eletrônicas www.tradeuk.com, www.tapin.co.uk e www.yell.com. Os questionários foram enviados para a totalidade da população (308 empresas), sendo que 5 voltaram com problemas de endereço, não sendo possível encontrar aquelas empresas.

A primeira remessa dos questionários foi enviada em meados de fevereiro de 2002 e a segunda remessa foi enviada em meados de março de 2002. Os sujeitos da pesquisa foram os Gerentes Industriais (Operacionais). Dos 303 questionários entregues 99 retornaram em condições de análise, com uma taxa de retorno de $32,67 \%$. De acordo com a revisão de literatura, é comum trabalhar-se com uma taxa de retorno entre $20 \%$ e $30 \%$ (Heide \& John, 1990; Kumar, Scheer, \& Seenkamp, 1995; Tan et al., 1999).

Para análise dos dados foi utilizado o pacote estatístico SPSS. O primeiro teste realizado tinha o objetivo de verificar se as 99 empresas respondentes representavam a população. Assim, realizou-se um teste qui-quadrado $\left(\chi^{2}\right)$, utilizando-se como variáveis o faturamento e a posição geográfica. Além disso, realizou-se o teste de confiabilidade da escala, chamado de Cronbach's Alfa $(\alpha)$.

Para testar a hipótese $\mathbf{H 1}$ utilizou-se a análise fatorial, sendo esta uma técnica estatística multivariada que tem por objetivo agrupar variáveis que estão altamente correlacionadas em conjuntos de variáveis chamados de fatores.

Como um dos pressupostos para análise fatorial Hair et al. (1998) recomenda que seja verificada a normalidade da variável e que haja pelo menos 5 observações para cada variável que compõe as questões, tendo no mínimo 100 respondentes. Além disso, Hair et al (1998) sugerem que para a aplicação da análise fatorial três premissas devem ser verificadas: 1-verificação de correlação entre as variáveis (BTS); 2- verificação da matriz de antiimagem de correlação. Hair et al. (1998), advogam que um pouco de colinearidade é desejado entre as variáveis, para que se realize a análise fatorial; 3- o teste Kaiser-Mayer-Olkin (KMO), que é uma medida de adequação da amostra.

Para extração dos fatores escolheu-se a análise dos componentes principais 
(Principal Components Analysis - PCA) por possuir características que atendiam à necessidade desta pesquisa. Na literatura foram identificados trabalhos que utilizaram o mesmo método com objetivos conceitualmente similares, podendo-se citar Monczka, Petersen e Handfield (1998); Tan et al. (1999) e Gundlach, Achroc e Mentzer (1995). A extração dos fatores foi realizada de forma a obter o máximo da variância possível. Segundo Hair et al. (1998) e Tabachnick e Fidell (1989), neste método cada variável contribui com pelo menos uma unidade para a variância total, chamada de autovalor e o critério mínimo aceitável indica que é necessário que cada fator tenha pelo menos uma unidade em seu autovalor. O resultado da análise fatorial é uma matriz inicial na qual se explicita o relacionamento. Entretanto a interpretação da matriz inicial não-rotacionada torna-se um pouco difícil. Diante do problema de interpretação dos resultados, o método prevê um processo que se chama de rotação. De acordo com Tabachnich e Fidell (1989), quando se rotaciona a matriz componente a variância é distribuída entre todos os fatores, o que conduz normalmente a um melhor agrupamento das variáveis de uma forma mais evidente, sendo que as correlações mais fortes são maximizadas e as mais fracas minimizadas. Estes autores ressaltam que a rotação ortogonal deve ser utilizada somente se os pesquisadores tiverem plena convicção da total independência dos fatores. Como esta pesquisa envolvia fatores de relacionamento entre fornecedores e compradores, decidiu-se pelo uso da outra forma de rotação, a rotação oblíqua. Na interpretação da matriz rotacionada, Hair et al. (1998) defendem que valores inferiores a 0,3 devem ser sumariamente desconsiderados na análise; valores maiores que 0,4 já podem ser considerados significantes; e os valores acima de 0,5 podem ser considerados realmente significantes.

Para o teste da hipótese $\mathbf{H 2}$ e $\mathbf{H 3}$ estabeleceu-se que seriam realizados os testes de Pearson qui-quadrado $\left(\chi^{2}\right)$ e ANOVA. Se o resultado desta análise indicasse o relacionamento entre as duas variáveis, far-se-ia, como complemento o método da regressão logística, a fim de aprofundar a análise. Segundo Sincich (1996) o método Pearson qui-quadrado $\left(\chi^{2}\right)$ tem por objetivo investigar o relacionamento entre duas variáveis, utilizando para isso variáveis categóricas, determinando se existe interdependência de duas variáveis qualitativas ou categóricas. A única condição inicial requerida para o teste $\chi^{2}$ é que a frequiência esperada para cada célula não pode ser pequena. Para realização deste teste a hipótese nula $H o$ foi estabelecida considerando-se que as duas direções (linhas e colunas) das tabelas de contingências eram independentes.

De acordo com Sincich (1996), a regressão logística binária é um bom método, quando a variável dependente tem apenas dois grupos. Além disso, esse tipo de regressão não se depara com restrições rígidas. A regressão logística utiliza como mecanismo de predição as probabilidades, sendo que apesar da probabilidade poder variar de 0 (zero) a 1 (um), os valores da predição não devem assumir 
estes valores extremos. Esta regressão fornece os coeficientes estimados para as variáveis independentes. A natureza não-linear da transformação logística requer o uso da máxima verossimilhança para encontrar os coeficientes estimados mais prováveis. De maneira geral, o antilogaritmo dos coeficientes $\beta_{i}$ nos modelos de regressão logística representa a chance de ocorrer $\{y=1\}$ quando $\boldsymbol{X}_{i}$ cresce em uma unidade, mantendo-se todos os demais $\boldsymbol{X}$ 's fixos, presentes no modelo. Alguns pesquisadores, frequientemente, calculam o coeficiente $\left[\left(\boldsymbol{e}^{\beta i}\right)-1\right]$ com o objetivo de obter o percentual de mudança na probabilidade de ocorrer um evento. Se os valores de $\left[\left(\boldsymbol{e}^{\beta i}\right)-1\right]$ forem positivos, haverá a seguinte implicação: as chances de o evento ocorrer aumentam; se for negativo, significa o oposto.

A análise da variância (ANOVA) foi utilizada para comparar as médias de grupos de respondentes. No caso específico desta pesquisa ela foi utilizada para comparar benefícios alcançados, nos diferentes grupos de investimentos feitos de capital e tempo gerencial dedicado. Quanto maiores as variações, maiores serão as evidências de que existe diferença nas médias das duas populações $\mu_{1} \mathrm{e}$ $\mu_{2 .}$ A hipótese nula Ho era que não existia diferença entre as médias populacionais.

\section{Limitações da Pesquisa}

As limitações desta pesquisa relacionaram-se diretamente com as escolhas que foram realizadas. Tais escolhas limitaram a possibilidade de generalização ampla e completa dos resultados. A primeira limitação referia-se ao escopo de investigação, estando restrita à cadeia de hortifrutigranjeiros frescos e minimamente processados. Além disso, deve-se destacar que a pesquisa foi realizada apenas no Reino Unido, não havendo, assim, a possibilidade de se estabelecer, neste momento, uma base mundial para a cadeia de hortifrutigranjeiros frescos. $\mathrm{O}$ segundo fator limitante da pesquisa estava no fato de as informações terem sido captadas em apenas um dos lados do relacionamento, ou seja, na perspectiva dos fornecedores. A estratégia de pesquisa amostral e as técnicas estatísticas utilizadas limitaram a possibilidade de se estabelecer uma relação de causalidade entre os fatos. O principal fator limitador da relação de causalidade residia na ocorrência da coleta simultânea das variáveis analisadas. O fato de ter sido enviado apenas um questionário para cada empresa também limitou a pesquisa, pois a resposta obtida é de um dos membros, e não de todo corpo gerencial. Dessa forma, podiam ser encontradas distorções das percepções individuais em relação ao posicionamento coletivo. A última limitação observada residia no fato de se ter realizado a pesquisa em um único momento, o que estabelecia a necessidade de se considerarem as respostas de acordo com o espectro de uma visão estática. 


\section{Análise dos Dados e Resultados}

O primeiro teste realizado foi o teste qui-quadrado $\left(\chi^{2}\right)$, para verificar se os 99 respondentes representavam a população de 308 empresas. O teste apresentou resultados satisfatórios, tanto para a dimensão de faturamento quanto para a dimensão de posição geográfica. O segundo passo foi verificar se as recomendações de Hair et al. (1998) com relação à normalidade das variáveis e ao número mínimo de respondentes e à razão do número de observações estavam sendo atendidas. $\mathrm{O}$ teste de normalidade foi realizado e nenhum problema foi encontrado. Além disso, considerando-se os 99 respondentes e a razão 6,6 (99/ 15), observou-se que as duas últimas recomendações eram plenamente atendidas.

Antes de iniciar o teste das hipóteses são apresentados os dados que caracterizam as empresas, com o objetivo de contextualizar a análise. Do total, $36,7 \%$ das empresas faturam, anualmente, menos de 40 milhões de reais, $21,4 \%$ faturam entre 44 e 100 milhões, 22,4\% faturam entre 101 e 200 milhões e 19,4\% faturam mais de 201 milhões. Considerando-se que no Reino Unido as empresas de grande porte são as que faturam mais de 201 milhões, vê-se que a grande maioria é de pequeno e médio porte. Cerca de $17 \%$ dos fornecedores possuem menos que cinco compradores, ou seja, poucos fornecedores têm clientes exclusivos ou quase exclusivos. Esses dados permitem inferir que a política de redução da base de fornecedores desenvolvida pelas grandes redes de supermercados não se encontra em sua fase avançada de implementação, uma vez que quase a metade dos fornecedores (44.4\%) tem mais de vinte compradores. O número de fornecedores com mais de 5 compradores-chaves intermediários ${ }^{(5)}$ é de $43 \%$. Esse é um elemento que normalmente dificulta a implementação das parcerias. $\mathrm{O}$ grande número de parcerias desenvolvidas simultaneamente pelos fornecedores aumenta, sobremaneira, a necessidade de investimentos de recursos financeiros e de tempo gerencial, recursos estes, normalmente escassos nas empresas.

\section{Testes das Hipóteses}

Hipótese H1: Os benefícios alcançados na cadeia de hortifrutigranjeiros no Reino Unido coincidem com aqueles identificados pela literatura.

Conforme descrito na seção 3, Hair et al. (1998) recomendam que sejam realizados cálculos da correlação entre as variáveis (BTS), a verificação da matriz de antiimagem de correlação e a medida de adequação da amostra $M A S$. Entretanto, antes de calcular o BTS, foi feita uma inspeção visual na tabela de correlação entre as variáveis, verificando-se que 78 das 105 correlações eram significantes em um nível de 5\%. A partir destes dados foi calculado o Bartlett's 
Test of Sphericity (BTS), observando-se que o nível de significância foi zero. Verificou-se o valor de 0,73 para o KMO, o que conduziu a não necessidade de análise da matriz de anti-imagem de correlação. Considerando a facilidade de cálculo desta rotina fez-se o teste, observando-se que nenhuma das correlações apresentava valores relevantes, o que confirmava a pré-condição para a realização da análise fatorial ${ }^{(6)}$. Na Tabela 1 , são apresentados os resultados dos KMOs para cada grupo de variáveis, podendo-se verificar a inexistência de problemas.

\section{Tabela 1: Teste das Pré-condições para Análise Fatorial}

\begin{tabular}{|c|c|c|c|c|}
\hline Variáveis componentes - teóricas & $\begin{array}{c}\text { Fator teórico } \\
\text { Benefícios }\end{array}$ & $\begin{array}{c}\text { No. de } \\
\text { variáveis }\end{array}$ & $\alpha$ & $\mathrm{KMO}$ \\
\hline $\begin{array}{l}\text { 4A) Menor custo de produção } \\
\text { 4B) Alta percentagem de sucesso no desenvolvimento de } \\
\text { novos produtos } \\
\text { 4C) Menores custos de armazenagem e transportes } \\
\text { 4D) Alta taxa de retorno sobre os ativos físicos } \\
\text { 4E) Menos perdas de produtos }\end{array}$ & $\begin{array}{c}\text { BE1-Eficiência } \\
\text { nos custos e uso } \\
\text { dos ativos }\end{array}$ & 5 & 0,7267 & 0,698 \\
\hline $\begin{array}{l}\text { 4F) Alta percentagem de produtos entregue no tempo certo } \\
\text { 4G) Tempo de ciclo reduzido } \\
\text { 4H) Maior flexibilidade na programação de produção } \\
\text { 4I) Menores níveis de estoques }\end{array}$ & $\begin{array}{c}\text { BE2-Melhoria } \\
\text { do serviço ao } \\
\text { consumidor }\end{array}$ & 4 & 0,7720 & 0,763 \\
\hline $\begin{array}{l}\text { 4J) Um mix de produtos que efetivamente atende às } \\
\text { necessidades dos consumidores finais } \\
\text { 4K) Melhor conhecimento das necessidades do consumidor } \\
\text { final }\end{array}$ & $\begin{array}{l}\text { BE3-Vantagens } \\
\text { de mercado }\end{array}$ & 2 & 0,6533 & 0,500 \\
\hline $\begin{array}{l}\text { 4L) Maiores volumes de vendas } \\
\text { 4M) Menor volatilidade nos preços } \\
\text { 4N) Maiores médias nos preços } \\
\text { 4O) Melhores margens de rentabilidade }\end{array}$ & $\begin{array}{c}\text { BE4- } \\
\text { Crescimento nas } \\
\text { vendas e } \\
\text { rentabilidade }\end{array}$ & 4 & 0,7335 & 0,657 \\
\hline
\end{tabular}

Antes de iniciar a análise fatorial, cabe destacar que, do ponto de vista conceitual, a redução nos níveis de estoque conduz à redução dos níveis de serviço de atendimento aos consumidores. Assim, pode parecer incoerente a participação da variável (4I) no fator melhoria de serviço ao consumidor, apresentada na Tabela 1. Não se pode esquecer que a presente pesquisa foi desenvolvida na cadeia de hortifrutigranjeiros frescos, que trabalha com produtos muito perecíveis. Sabe-se que um dos atributos mais importantes de qualidade destes produtos é que estejam sempre frescos. Uma das formas de garantir produtos frescos é reduzir o tempo entre colheita e disposição na prateleira. Uma das formas de se conseguir esta redução é ter menores estoques de produtos pós-colheita. Esta observação se contrapõe, em parte, ao conceito de que maiores níveis de estoque conduzem a melhores níveis de serviço, por causa do risco de perda da qualidade do produto, em um curto espaço de tempo. 
Iniciando a análise fatorial, observou-se que foram extraídos cinco fatores. Esse resultado não confirmava os quatro fatores teóricos. Entretanto observouse que a variável $4 \mathrm{E}$ apresentava-se sozinha no último fator extraído. Em se tratando de análise fatorial e, de acordo com o método, quando isso ocorre devese tentar uma solução na qual se estabelece o número de fatores do último resultado alcançado menos um. Neste caso foi estabelecido que o resultado deveria gerar quatro fatores. É importante ressaltar que, neste caso específico, o critério de parada do método não foi definido em função do autovalor, mas em função do número de fatores extraídos.

Na Tabela 2 é apresentado o resultado da segunda análise fatorial. Verificase que a variável 4E passou a compor o fator BE1 Eficiência nos custos e uso dos ativos. Como a variável $4 \mathrm{E}$ representa redução nas perdas dos produtos e o seu coeficiente é pouco representativo em relação aos outros três que compõem o fator, decidiu-se manter esta solução como a solução final para a análise fatorial.

\section{Tabela 2: Resultado Final da Análise Fatorial para os Benefícios}

\begin{tabular}{|c|c|c|c|c|c|}
\hline \multirow{2}{*}{ Benefícios } & \multirow{2}{*}{ Variáveis } & \multicolumn{4}{|c|}{ Fatores } \\
\hline & & 1 & 2 & 3 & 4 \\
\hline \multirow{4}{*}{ BE1-Eficiência nos custos e uso dos ativos } & $4 \mathrm{~A}$ & & & 0,859 & \\
\hline & $4 \mathrm{C}$ & & & 0,728 & \\
\hline & $4 \mathrm{~B}$ & & & 0,711 & \\
\hline & $4 \mathrm{E}$ & & & 0,569 & \\
\hline \multirow{4}{*}{ BE2-Melhoria do serviço ao consumidor } & $4 \mathrm{H}$ & 0,825 & & & \\
\hline & $4 \mathrm{~F}$ & 0,821 & & & \\
\hline & $4 \mathrm{G}$ & 0,766 & & & \\
\hline & 4I & 0,722 & & & \\
\hline \multirow{3}{*}{ BE3-Vantagens de mercado } & $4 \mathrm{~K}$ & & & & 0,875 \\
\hline & $4 \mathrm{~J}$ & & & & 0,775 \\
\hline & $4 \mathrm{~L}$ & & & & 0,546 \\
\hline \multirow{4}{*}{ BE4-Crescimento nas vendas e rentabilidade } & 40 & & 0,878 & & \\
\hline & $4 \mathrm{~N}$ & & 0,875 & & \\
\hline & 4D & & 0,682 & & \\
\hline & $4 \mathrm{M}$ & & 0,666 & & \\
\hline Eigenvalores & & 4,579 & 2,135 & 1,426 & 1,237 \\
\hline \% da variância explicada & & 30,527 & 14,237 & 9,507 & 8,246 \\
\hline \% da variância acumulada explicada & & 30,527 & 44,764 & 54,271 & 62,516 \\
\hline
\end{tabular}


Do ponto de vista conceitual não havia problemas em assumir esta solução, uma vez que a redução de perdas pode ser conseguida com melhor previsão de demanda e melhoria na comunicação com os compradores intermediários. Com relação à estatística, essa solução era representativa, pois as variáveis envolvidas no modelo representam 62,51 \% da variância acumulada. De acordo com a bibliografia, um resultado superior a 50-60\% é relevante. Assim sendo, os Benefícios identificados pelos fornecedores de hortifrutigranjeiros do Reino Unido eram os mesmos identificados na literatura.

Hipótese H2: as empresas que estão investindo maior volume de capital nos relacionamentos com seus compradores-chaves intermediários estão obtendo mais benefícios que as empresas que investem pouco.

As duas variáveis envolvidas neste teste eram volume de investimento de capital em seus relacionamentos (variável categórica) e benefícios alcançados pelas empresas. Desta forma, tanto a variável volume de investimento de capital quanto a variável benefícios alcançados possuíam duas categorias. No primeiro caso, no nível zero (0) estavam as empresas que investiram menos de $50 \%$ do seu total de investimentos na melhoria das parcerias e, no nível (1) as empresas que investiram mais de $50 \%$ dos investimentos totais. A variável benefícios alcançados era representada por nível (0) benefícios não positivos (neutros e negativos), ou seja, consideravam que não obtiverem benefícios com as parcerias e, no nível (1) obtiveram benefícios positivos.

Conforme pode ser observado na Tabela 3, com significância de 5\%, o teste falhou em rejeitar Ho, para $\mathbf{H 2}$, ou seja, as duas variáveis eram independentes, não havendo assim nenhuma correlação entre elas. Esta hipótese estabelecia que os fornecedores que estavam colocando maior volume de capital em suas parcerias estariam obtendo maiores benefícios com isto. Entretanto não foram verificadas evidências para apoiar essa hipótese, ou seja, não havia evidencias estatísticas que apoiassem a hipótese $\mathbf{H 2}$ apresentada anteriormente.

Tabela 3: Teste de Qui-quadrado para Hipótese H2

\begin{tabular}{|c|c|c|}
\hline \multirow{2}{*}{ Variáveis } & \multicolumn{2}{|c|}{ Pearson qui-quadrado } \\
\cline { 2 - 3 } & Valor & Sig. \\
\hline $\begin{array}{c}\text { Investimento de capital } \\
\text { Benefícios alcançados }\end{array}$ & 2,297 & 0,13 \\
\hline
\end{tabular}

Para o teste ANOVA foram realizados, inicialmente, os testes dos pressupostos de normalidade e homocedasticidade da variável benefícios alcançados, não se observando quebra dos pressupostos. A partir dos dados apresentados na Tabela 
4, observou-se que se falhava em rejeitar a hipótese nula Ho: não existindo diferença entre as médias populacionais. Desta forma não se podia afirmar que existia diferença nos benefícios alcançados pelo grupo das empresas que estavam investindo pouco das empresas que investiam muito, reforçando assim a conclusão do teste qui-quadrado para a hipótese $\mathbf{H 2}$.

\section{Tabela 4: ANOVA dos Benefícios em Relação ao Capital Investido}

\begin{tabular}{|c|c|c|c|}
\hline \multicolumn{4}{|c|}{ ANOVA } \\
\hline & Média dos quadrados & F & Sig. \\
\hline Entre grupos MST & 0,836 & 3,294 & 0,073 \\
Dentro dos grupos MSE & 0,254 & & \\
\hline
\end{tabular}

Uma das explicações para a não dependência destas duas variáveis pode estar na dificuldade técnica de os fornecedores identificarem a quantidade de recursos dedicados a cada investimento especifico. Por exemplo, é difícil alocar, de forma detalhada, os investimentos em softwares e pesquisa e desenvolvimento, realizados pelos fornecedores, dedicados exclusivamente a cada um dos seus parceiros.

Hipótese H3: As empresas que estão investindo maior tempo gerencial nos relacionamentos com seus compradores-chaves intermediários estão obtendo mais benefícios que as empresas que investem pouco.

As duas variáveis envolvidas neste teste eram investimento de tempo gerencial em seus relacionamentos (variável categórica) e benefícios alcançados pelas empresas. A variável investimento de tempo gerencial assumia valor zero (0), quando a empresa investia valores menores ou iguais a 50\% do total; e nível (1), quando investia valores maiores que 50\%. A variável benefícios assumia nível (0), quando os benefícios eram não positivos (neutros e negativos); e nível (1) quando os benefícios eram positivos.

Na Tabela 5 observou-se que Ho deveria ser rejeitada com significância de 5\%. Assim, era possível afirmar que as duas variáveis eram dependentes, havendo correlação entre elas. Desta forma, a tendência do coeficiente (sinal dos $\beta s$ ) e a magnitude da dependência (relevância dos $\beta s$ ) deveriam ser verificados, utilizando-se o teste de regressão logística (binária). Para o teste de regressão logística o SPSS estabelece como padrão a hipótese nula Ho: os coeficientes $\beta \mathbf{s}$ são zero. 
Tabela 5: Teste de Qui-quadrado para hipótese H3

\begin{tabular}{|c|c|c|}
\hline \multirow{2}{*}{ Variáveis } & \multicolumn{2}{|c|}{ Pearson qui-quadrado } \\
\cline { 2 - 3 } & valor & Sig. \\
\hline $\begin{array}{c}\text { investimento em tempo gerencial } \\
\text { benefícios alcançados }\end{array}$ & 7,646 & 0,05 \\
\hline
\end{tabular}

\section{Tabela 6: Resultado da Regressão Logística para hipótese H3}

\begin{tabular}{|c|c|c|c|}
\hline Coeficiente $\beta$ & Significância. & Constante & Nagelkerke $\mathrm{R}^{2}$ \\
\hline 2,413 & 0,022 & $-2,708$ & 0,126 \\
\hline
\end{tabular}

De acordo com o resultado apresentado verificou-se que o coeficiente $\beta$ possuía sinal positivo, ou seja, as duas variáveis eram positivamente correlacionadas. $\mathrm{O}$ sinal positivo de $\beta$ produziu um valor positivo de [( $\left.\left.\boldsymbol{e}^{\beta i}\right)-1\right]$. Sincich (1996) destaca que o valor positivo de $\left[\left(\boldsymbol{e}^{\beta i}\right)-1\right]$ implica, obrigatoriamente, o crescimento da probabilidade de ocorrência e um valor negativo implica, necessariamente, a diminuição da probabilidade de ocorrência.

Diante disso, era possível estabelecer que para a hipótese $\mathbf{H 3}$ os fornecedores que investiram positivamente em seu tempo gerencial na melhoria do seu relacionamento com os parceiros estavam obtendo mais benefícios que os que não o faziam. Em outras palavras, a probabilidade de conseguir os benefícios das parcerias era $10\left\{\left[\left(\boldsymbol{e}^{\beta i}\right)-1\right]=10,16\right\}$ vezes superior para os fornecedores que investiam positivamente seu tempo gerencial, do que para aqueles que não o faziam.

No teste de ANOVA a hipótese nula estabelecida foi Ho: não existe diferença entre as médias populacionais. De posse dos resultados apresentados na Tabela 7, verificou-se que a hipótese nula foi rejeitada com significância de $0,8 \%$, ou seja, era possível afirmar que existia diferença nos "benefícios alcançados" pelo grupo das empresas que estavam investindo pouco tempo gerencial daquelas empresas que investiram muito, confirmando, assim, a hipótese H3.

\section{Tabela 7: ANOVA dos Benefícios em Relação ao Tempo Gerencial Investido}

\begin{tabular}{|c|c|c|c|}
\hline \multicolumn{4}{|c|}{ ANOVA } \\
\hline & Média dos quadrados & F & Sig. \\
\hline Entre grupos MST & 1,767 & 7,283 & 0,008 \\
Dentro dos grupos MSE & 0,243 & & \\
\hline
\end{tabular}


Diferentemente da hipótese $\mathbf{H 2}$, na qual foi considerada a dificuldade de se identificarem os investimentos financeiros, nesse caso era muito mais fácil para o fornecedor identificar o tempo dedicado a cada um dos parceiros. Pode-se inferir, então, que os fornecedores de hortifrutigranjeiros do Reino Unidos estão, atualmente, em um estágio avançado dentro de suas parcerias. É natural que, em um primeiro momento, as empresas invistam em tempo, porém os resultados não aparecem rapidamente. Desta forma, pode-se considerar o fato de já estarem obtendo os benefícios, um estágio avançado.

\section{Conclusões e Sugestões}

De acordo com Demo (2000) a pesquisa empírica contribui para a expansão das fronteiras do conhecimento, abordando a realidade pelo prisma empírico, indo além do que aparece à primeira vista. Desta forma, esta pesquisa contribui para aumentar o conhecimento, por meio da produção e análise dos dados empíricos, considerandose a escassez de pesquisas empíricas nesta área do conhecimento.

Os resultados dos testes das hipóteses demonstraram haver evidências estatísticas para confirmar as hipóteses H1 e H3. Entretanto não foram encontradas evidências, estatisticamente significantes, para apoiar as hipóteses H2. Em outras palavras, para a hipótese $\mathbf{H 3}$ houve correlação entre as variáveis, e para a hipótese $\mathbf{H} 2$ não houve correlação.

Uma das preocupações gerenciais desta pesquisa concentrou-se na possibilidade de obtenção dos benefícios advindos das parcerias por parte dos fornecedores. Apesar de não ser objeto de análise do presente artigo, durante a pesquisa no Reino Unido, identificou-se que na cadeia de hortifrutigranjeiros os compradoreschaves intermediários, de forma mais específica as grandes redes de supermercados, são o elo mais forte na negociação. Assim, extrapolando a análise para além dos resultados, observou-se que os fornecedores, sendo o elo mais fraco do relacionamento, têm a possibilidade de alcançar os benefícios das parcerias, aumenta, sobremaneira, a possibilidade do relacionamento denominado ganha-ganha. Esse estilo de relacionamento pode criar condições necessárias para que o GCS seja colocado em prática, uma vez que aumenta as potencialidades das parcerias de sucesso.

A não-confirmação da hipótese $\mathbf{H 2}$ remete à necessidade de se aprimorarem, atualmente, os sistemas de informação relativos ao monitoramento dos investimentos feitos nas parcerias. Um dos grandes problemas gerenciais no início das parcerias é identificar quais investimentos financeiros foram realizados para 
a melhoria do relacionamento. Em outras palavras, para que os fornecedores decidam se é benéfico para eles estabelecer parcerias, é necessário que os investimentos sejam identificados, e isso não estava ocorrendo.

Outro fato gerencial relevante identificado foi a confirmação da hipótese H3, na qual os fornecedores que estavam dedicando maior tempo gerencial às suas parcerias têm obtido maiores benefícios nelas. Apesar de não se ter investigado empiricamente os fatores intangíveis, os autores deste artigo consideram que o tempo gerencial tem papel fundamental para a melhoria dos fatores intangíveis. Em princípio, com maior tempo dedicado ao relacionamento haveria maior possibilidade de se gerenciarem as assimetrias do relacionamento e aumentando a confiança do parceiro. Conclui-se, então, que dedicar tempo à parceria é fundamental para a obtenção de bons resultados.

As conclusões do presente artigo possibilitam a continuidade da pesquisa iniciada, bem como a ampliação do seu escopo de atuação. Diante desse fato, são indicadas as seguintes alternativas:

- Desenvolver pesquisa nas cadeias de alimentos frescos em outras partes do mundo, a fím de criar uma base mundial (projeto já em andamento). Além disso, esta pesquisa possibilitará a comparação da cadeia britânica de alimentos com outras cadeias, já que ela é considerada referência mundial.

- Pesquisar cadeias com produtos inovadores, a fim de criar uma possibilidade de comparação com a cadeia de alimentos frescos, caracterizados como produtos funcionais (commodities).

- Replicar a pesquisa no Reino Unido com respeito ao relacionamento entre os fornecedores e seus fornecedores de primeiro nível, com o objetivo de comparar os elementos do relacionamento identificados.

\section{Artigo recebido em 23.06.2004. Aprovado em 14.09.2004.}

\section{Notas}

\footnotetext{
${ }^{1}$ Os autores deste artigo agradecem o apoio financeiro recebido da CAPES e da FAESA. Além disso, agradecemos a contribuição dos avaliadores.

${ }^{2}$ As pessoas que desejarem obter o questionário poderão fazer contato com o primeiro autor deste artigo.

${ }^{3} \mathrm{Na}$ presente pesquisa não foram identificados os produtos comercializados, nem as suas origens. Estas duas dimensões não foram consideradas, porque não interferem no gerenciamento dos relacionamentos dentro da cadeia de abastecimento.
} 
${ }^{4}$ Foi considerado o câmbio de 4 reais por libra esterlina (realidade na época da pesquisa).

${ }^{5} \mathrm{O}$ conceito de comprador intermediário chave foi utilizado para identificar como os fornecedores conceituam suas parcerias, uma vez que o conceito de parceria é muito amplo. Por não ser objeto principal deste artigo este assunto será tratado, oportunamente, em outro artigo.

${ }^{6}$ A tabela de antiimagem não é apresentada aqui por limitação de espaço.

\section{ReferênCIAS Bibliográficas}

Bhatnagar, R., \&

Viswanathan, S. (2000, February).

Re-engineering global supply chains alliances between manufacturing firms and global logistics services providers. International Journal of Physical Distribution \& Logistics Management, 30(1), 13-34.

Bowersox, D. J.,

Closs D. J., \&

Keller, S. B. (2000, September/October). How supply chain competency leads to business success. Supply Chain Management Review, 4(4), 70-78.

Cavinato, J. L. (1992, July/December). A total cost/value model for supply chain competitiveness. Journal of Business Logistics, 13(2), 285-301.

Cooper, M. C.,

Ellram, L. M., \&

Gardner, J. T. (1997, July/December). Meshing Multiple Alliances. Journal of Business Logistics, 18(1), 67-89.

Demo, P. (2000). Metodologia do conhecimento científico. São Paulo: Atlas.

Fearne, A., \& Hughes, D. (1998).

Success factors in the fresh produce supply chain: some examples from the UK. Food Industry Management Report, Wye College, University of London.

Fearne, A., \&

Hughes, D. (2000, November).

Success factors in the fresh produce supply chain. British Food Journal, 102 (10), 760-772.

Food Traders Directory of the UK and Europe (2000).

Fresh Produce (28th ed., Vol.1). London: UK, Ed. J. Wheatley, Newman Books.

Gunasekaran, A.,

Patel, C., \&

Tirtiroglu, E. (2001, January/February).

Performance measures and metrics in a supply chain environment. International Journal of Operations \& Production Management, 21(1/2), 71-87.

Gundlach, G. T., Achroc, R. S., \&

Mentzer, J. T. (1995, January).

The structure of commitment in exchange. Journal of Marketing, 59(1), 78-92. 
Hair, J. F., Jr.,

Anderson, R. E.,

Tatham, R. L., \&

Black, W. C. (1998).

Multivariate data analysis with readings (5th ed.). New Jersey, U.S.A: Prentice-Hall.

Hall, R., \&

Andriani, P. (1998, December).

Analysing Intangible Resources and Managing Knowledge in a Supply Chain Context. European Management Journal, 16(6), 685-697.

Heide, J., \&

John, G. (1990, February).

Alliances in Industrial Purchasing: The Determinants of Joint Action in BuyerSupplier Relationships. Journal of Marketing Research, 27(1), 24-36.

Lambert, D. M.,

Emmelhainz, M. A., \&

Gardner, J. T. (1996, July/December).

Developing and Implementing Supply Chain Partnerships. The International Journal of Logistics Management, 7(2), 1-17.

Keah-Choon, T,

Kannan, V. R.,

Handfield, R. B., \&

Soumen G. (1999, October).

Supply chain management: an empirical study of its impact on performance. International Journal of Operations \& Production Management, 19(10), 1034-1052.

Kumar, N.,

Scheer, L. K., \&

Steenkamp, E. M. (1995, August).

The effects of perceived
Interdependence on dealer attitudes. Journal of Marketing Research, 32(3), 348-356.

Maloni, M. J., \&

Benton, W. C. (1997, September).

Supply chain partnerships: Opportunities for operations research. European Journal of Operational Research, 101(3), 419-429.

Monczka, R. M.,

Petersen, K. J., \&

Handfield, R. B. (1998, Summer).

Success Factors in Strategic Supplier

Alliance: the Buying Company Perspective. Decision Science, 29(3), 553-577.

Noordewier, T. G.,

John, G., \&

Nevin, J. R. (1990, October/December).

Performance Outcomes of Purchasing Arrangements in Industrial BuyerVendor Relationships. Journal of Marketing, 54(4), 80-93.

Oliver, C. (1990, April).

Determinants of inter-organisational relationships: integration and future directions. Academy of Management, 15(2), 241-265.

Plimsoll Portfolio Analysis (1999)

Fresh Produce, Main Report, Parts $1 \& 2$ (2nd ed.). Middlesbrough, U.K.: Plimsoll Publishing Limited.

Sandy, J. D. (1999, November/January).

Pie-Expansion Efforts: Collaboration Processes in Buyer-Supplier Relationships. Journal of Marketing Research, 36(4), 461-475. 
Sincich, T. (1996).

Business statistics by example (5th ed.). New Jersey, USA: Prentice-Hall.

Stank, T., \&

Crum, M. (1999, July/December).

Benefits of interfirm co-ordination in food industry supply chain. Journal of Business Logistics, 20(2), 21-41.

Tabachnick, B. G., \&

Fidell, L. (1989).

Using multivariate statistics (2nd ed.). New York, USA: Harper-Collins Publishers.
Van Hoek, R. I. (1998, October/December). Measuring the unmeasurable measuring and improving the performance in the supply chain. Supply Chain Management, 3(4), 187192.

Vergara, S. (1998).

Projetos e Relatórios de Pesquisa em Administração (2a ed.). São Paulo: Atlas. 